\title{
Congenital absence of superior vena cava. Case report and review of the literature
}

\author{
Mihaela Demetrian', Andreea Vidru', Alexandra Cozinov' ${ }^{1}$, Cristina Dumitru', \\ Andreea Avramescu', Veronica Marcu ${ }^{2}$, Andra Pirnuta', \\ Alexandra Bouariu', Anca Panaitescu ${ }^{1,3}$ \\ ${ }^{1}$ Department of Neonatology, Filantropia Clinical Hospital, Bucharest, Romania \\ 2Department of Radiology, "Marie Curie Sklodowska” Emergency Clinical Hospital for Children, \\ Bucharest, Romania \\ 3"Carol Davila" University of Medicine and Pharmacy, Bucharest, Romania
}

\begin{abstract}
Superior vena cava (SVC) agenesis or total congenital absence of superior vena cava is a very rare vascular anomaly, mainly asymptomatic throughout neonatal period. We present a case of bilateral total congenital absence of superior vena cava and thoracic duct obstruction in a newborn which were detected during the anomaly scan at 22 weeks gestation as unilateral pleural effusion. Transthoracic echocardiography and angiography computed tomography in the postnatal period clearly established the diagnosis of congenital anomaly of the great veins. The pleural lymphatic effusion has resolved after drainage and medical treatment in 14 days.
\end{abstract}

Keywords: congenital chylothorax, agenesis of superior vena cava

\section{INTRODUCTION}

Bilateral absence of superior vena is a unique form of superior vena cava anomalies. Mainly asymptomatic and usually found incidentally the agenesis of superior vena cava is poorly reviewed in the literature, and therefore the incidence in the general population may be higher than previously reported. Bilateral superior vena cava agenesis occurs in the general population up to $0.3 \%$ in comparison with patients with associated congenital heart disease with an incidence of $3 \%$ to $11 \%$ [1]. Among clinical findings related to this vascular anomaly, cystic hygroma during early intrauterine period and neonatal chylothorax are the most common.

Neonatal chylothorax is defined as the accumulation of lymphatic fluid in the pleural cavity and can be congenital or acquired [2-5]. Congenital chylothorax can be idiopathic or it may be associated with chromosomal abnormalities, including Down syndrome [2,5,6], Turner syndrome [7], Noonan syndrome [8,9] and other genetic disorders [10-13]. Congenital pulmonary lymphangiectasia and gener- alized lymphangiomatosis are also associated with congenital chylothorax [14-16].

\section{CASE REPORT}

A 29-year-old primipara was examined in our U1trasound Department for the first trimester assessment and the sonographic evaluation showed an increased nuchal translucency $(3.74 \mathrm{~mm})$. Amniocentesis was carried out and the analyses of QF-PCR and Microarray established a normal fetal karyotype. The second trimester ultrasound (US) performed at 22 weeks showed a slightly increased nuchal fold $(6.14 \mathrm{~mm})$, mild unilateral pleural effusion $(2-3 \mathrm{~mm})$, without superior vena cava (SVC) syndrome. Fetal cardiac assessment showed no obvious defect of the heart and great vessels, although there was a persistent reversed flow in the ductus venosus. Further testing was performed with negative results of the most common infections associated with congenital anomalies (TORCH panel, including parvovirus B19).

Later on, during pregnancy, the US assessment in the third trimester revealed significant left sided pleu- 
ral effusion $(11 / 44 / 52 \mathrm{~mm})$ with marked right mediastinal shift. The flow in the ductus venosus was normal and the azygos vein appeared mildly enlarged.

The infant was delivered by Caesarean section at 39 weeks, without peri- and intraoperative complication. The birthweight was $3700 \mathrm{~g}$ and the APGAR score 7 and 8 at 1 and 5 min. Physical examination of the newborn revealed swelling of the face, neck (lateral cervical and submandibular) and upper body; the head circumference was plotted above $90^{\text {th }}$ centile. Immediately after delivery, the newborn developed signs of acute respiratory distress syndrome that required intubation and high frequency ventilation with VG-mode activated ventilation. Intratracheal administration of surfactant and inhaled nitric oxide (INO) for 48 hours have been added to moderate the respiratory distress syndrome. Radiographs of the thorax showed massive left sided pleural effusion and pneumothorax with marked right mediastinal shift (Figures 1 and 2). A sample of $50 \mathrm{ml}$ serous pale-yellow pleural fluid was firstly drained through thoracocentesis. After starting enteral feeding, chylous pleural effusion was drained and analyzed, showing $1,280 \mathrm{mg} / \mathrm{dl}$ triglyceride and microscopic examination revealed $93 \%$ lymphocytes.

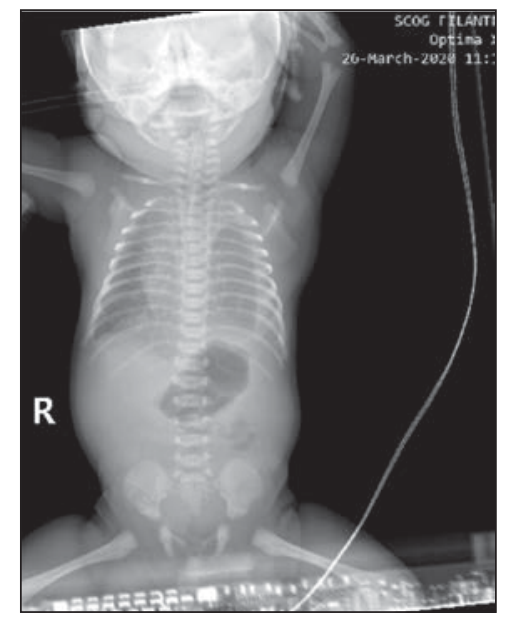

FIGURE 1. Chest

$X$-ray showing massive left sided pleural effusion

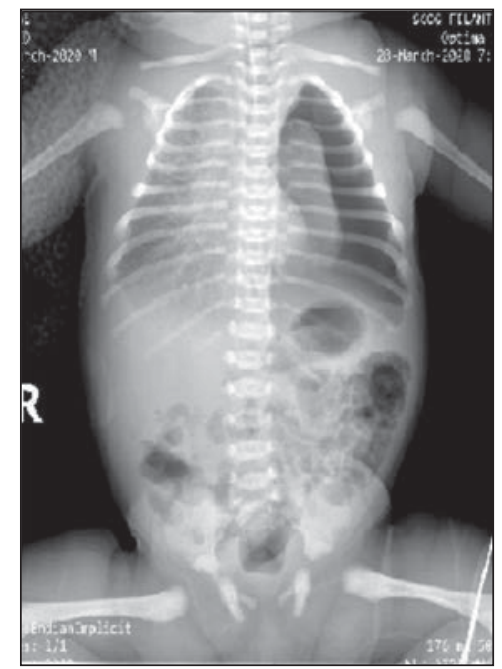
FIGURE 2. Chest X-ray showing pneumothorax with marked right mediastinal shift
The results of the computed tomography (CT) angiography showed enlargement of azygos vein between the arch that empties into the superior vena cava and the level of the vertebral root T10 and a normal size diameter below that level with dilation of vertebral vein that continues the azygos. The upper body venous system drained via azygos and hemiazygos veins into brachiocephalic vein. Inferior vena cava was visible only on the supra- and retrohepatic segment. Abdominal and cerebral vascular assessment was deemed to be normal (Figure 3).

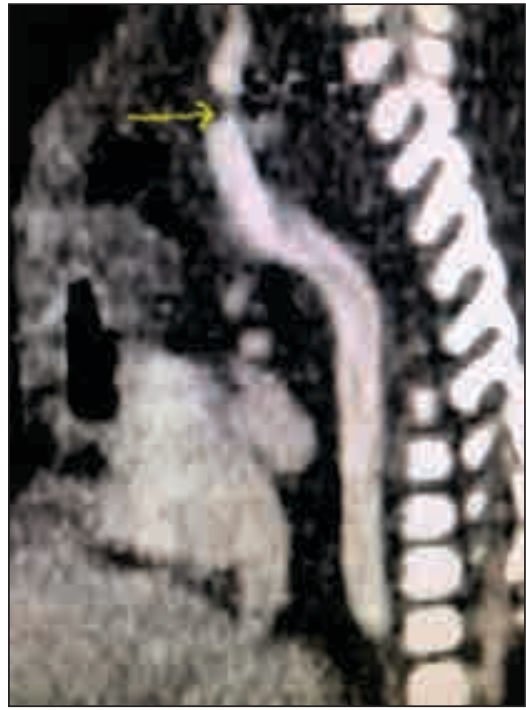

FIGURE 3. Angio-CT

The preliminary diagnosis of SVC syndrome due to thrombosis, therefore mechanical obstruction of the blood flow to the right atrium of the heart through SVC, was raised. The suspicion was immediately ruled out through laboratory common coagulation tests and lipid profile that showed normal values. Other metabolic causes of chylothorax were investigated, such as thyroid dysfunction, but the specific hormones suggested normal levels.

The newborn was intubated and ventilated for 14 days, with 3 additional pleural drainage required to alleviate the respiratory function. The results of biochemistry and microscopic investigations were similar to the previous one. The respiratory distress syndrome has been improved and the amount of pleural fluid decreased after administration of Octreotide 5 $\mu \mathrm{g} / \mathrm{kg} / \mathrm{h}$ for 11 days and replacement of breast milk with infant milk formula containing synthetic medium-chain triglycerides. The newborn was discharged after 33 days of care with the recommendation of keeping the same milk formula.

The clinical assessment by 2 months of age showed a good general condition, a mild respiratory distress syndrome with oxygen saturation of $96 \%$ and no other significant respiratory signs. The abdomen 
was soft and no signs of hepatosplenomegaly were noted. The brain development was normal for age. An additional radiography showed normal thoracic aspect with no pleural effusion. Similarly, the echocardiography revealed normal aspect of venous drainage with no enlargement and a normal aortic arch. There was a mild left to right interatrial shunt, with intact interventricular septum.

\section{DISCUSSIONS}

Suspicion of superior vena cava agenesis may arise during fetal routine echocardiography, as happened in the presented case. Perinatally investigations such as postnatal echocardiography, radiography and conventional angiography should follow in case of any unexplained clinical findings such as pleural effusion.

Congenital chylothorax results due to abnormal development or obstruction of the vessels from the lymphatic system. It is usually associated with hydrops fetalis $[2,4,5]$. Case report series described the possibility of familial congenital chylothorax recurrence, which may reveal a familial genetic transmission [16-19].

The mortality rate, as several case report series showed, is between 30 and $50 \%[5,20]$. The majority of the cases have been prenatally diagnosed and intrauterine interventions (such as, thoracic drainage and intra-amniotic thoracic shunt) were performed in $40 \%$ of cases. Preterm birth is considered a risk factor for death. Some studies show that the resolution of hydrops fetalis after intra-amniotic shunt improves significant the survival rate [21].

Cardiovascular malformations have been associated with non-immune hydrops fetalis NIHF in $40 \%$ of cases [22]. Congenital heart conditions (e.g., structural cardiac disorders and heart arrhythmias) that can lead to cardiac insufficiency, may be associated with pleural effusion and hydrops fetalis [2,5,21]. Pleural effusion was described in various case series with bilateral superior vena cava agenesis [23], glomuvenous malformation [24] and placental chorioangioma [21].

Unilateral SVC agenesis is mainly asymptomatic (in the vast majority of the cases it is an incidental finding) and usually the heart structure is normal. Bilateral agenesis is frequently associated with congenital cardiac abnormalities (tetralogy of Fallot, atrial septal defect) and arrhythmias, with SVC syndrome manifestations. The most common SVC anomaly is bilateral SVC, therefore both superior right and left vena cava are present. Not only bilateral SVC, but also isolated left SVC can be a normal variant or can be associated with cardiac congenital abnormalities
[2]. If isolated left SVC drains into right atrium, the venous drainage is normal. As a result, this abnormality is asymptomatic and the finding is incidentally. Absence of bilateral SVC is specific to SVC anomalies.

To our knowledge, there are just a few cases of agenesis of superior vena cava described in the literature [25]. Some cases were diagnosed in adults by venography during the procedure of pacemaker implantation [26] or electrophysiological evaluation for ablation purpose [27]. Other cases have been diagnosed in fetuses with associated cardiac abnormalities such as tetralogy of Fallot [28] and atrial septal defect [29].

Similar with the present case there are 2 other case reports in the literature that describe newborns suffering from chylothorax, one diagnosed prenatally and one postnatally [30].

Other cases have reported abnormal vascular azygos veins drainage into the left renal vein or into right atrium [27-29]. The clinical findings of bilateral superior vena cava agenesis are generally poor in normal fetuses or are associated with other cardiac or vascular abnormalities. Most common the diagnosis is described during routine fetal assessment or related to postnatally cardiac procedures.

In our case, we suppose that chylothorax was related to absence of superior vena cava and abnormal lymphatic drainage. After the connection of the new lymphatic vessels to the venous system, the chylothorax has dissolved and the outcome of the newborn was favorable. Further research is needed to improve the diagnosis and management in patients with bilateral superior vena cava agenesis.

\section{CONCLUSIONS}

Superior vena cava agenesis is a very rare anomaly, characterized by complete unilateral or bilateral superior vena cava absence. Because of its incidental finding and unspecific clinical appearance, the incidence may be higher than already reported in the literature. However, when associated with cardiac and other vascular abnormalities may complicate cardiac and central venous catheterization, temporary cardiac pacing or cardiac surgery. The possibility of this rare superior vena cava anomaly and the association with cardiac abnormalities should also be kept in mind when unexplained pleural effusion as well as septated cystic hygroma, even spontaneously resolved during fetal life, arise. The neonatology team and fetal medicine specialits should be aware of and well informed about all fetal findings diagnosed thoroughout intrauterine period in order to improve the management and fetal outcome. 


\section{REFERENCES}

1. Buirski G, Jordan SC, Joffe HS, Wilde P. Superior vena caval abnormalities: their occurrence rate, associated cardiac abnormalities and angiographic classification in a paediatric population with congenital heart disease. Clin Radiol. 1986;37(2):131-8.

2. Bellini $\mathrm{C}$, Boccardo $F$, Bonioli $E$, Campisi C. Lymphodynamics in the fetus and newborn. Lymphology. 2006;39(3):110-7.

3. Hansen KC, D'Alessandro A, Clement CC, Santambrogio L. Lymph formation, composition and circulation: a proteomics perspective. Int Immunol. 2015;27(5):219-27.

4. Cooper LJ, Heppell JP, Clough GF, Ganapathisubramani B, Roose T. An Image-Based Model of Fluid Flow Through Lymph Nodes. Bull Math Biol. 2016;78(1):52-71.

5. Bellini C, Ergaz Z, Boccardo F, Bellini T, Campisi CC, Bonioli E, Ramenghi LA. Dynamics of pleural fluid effusion and chylothorax in the fetus and newborn: role of the lymphatic system. Lymphology. 2013;46(2):75-84.

6. Downie L, Sasi A, Malhotra A. Congenital chylothorax: associations and neonatal outcomes. J Paediatr Child Health. 2014;50(3):234-8.

7. Bialkowski A, Poets CF, Franz AR; Erhebungseinheit für seltene pädiatrische Erkrankungen in Deutschland Study Group. Congenital chylothorax: a prospective nationwide epidemiological study in Germany. Arch Dis Child Fetal Neonatal Ed. 2015;100(2):F169-72.

8. Bellini C, Hennekam RC, Boccardo F, Campisi C, Serra G, Bonioli E. Nonimmune idiopathic hydrops fetalis and congenital lymphatic dysplasia. Am J Med Genet A. 2006;140(7):678-84.

9. Brock WW, Bradshaw WT. Congenital Chylothorax: A Unique Presentation of Nonimmune Hydrops Fetalis in a Preterm Infant. Adv Neonatal Care. 2016;16(2):114-23.

10. Bellini C, Mazzella M, Campisi C, Taddei G, Mosca F, Tomà P, Villa G, Boccardo F, Sementa AR, Hennekam RC, Serra G. Multimodal imaging in the congenital pulmonary lymphangiectasia-congenital chylothorax-hydrops fetalis continuum. Lymphology. 2004; 37(1):22-30.

11. Rocha G, Fernandes P, Rocha P, Quintas C, Martins T, Proença E. Pleural effusions in the neonate. Acta Paediatr. 2006 Jul;95(7):791-8.

12. Faul JL, Berry GJ, Colby TV, Ruoss SJ, Walter MB, Rosen GD, Raffin TA. Thoracic lymphangiomas, lymphangiectasis, lymphangiomatosis, and lymphatic dysplasia syndrome. Am J Respir Crit Care Med. 2000;161(3 Pt 1):1037-46.

13. Römer S, Opgen-Rhein B, Chaoui R, Scheer I, Czernik C, Obladen M. Bilateral agenesis of the superior vena cava associated with congenital hydrothorax. Ultrasound Obstet Gynecol. 2006; 28(6):842-4.

14. Bellini C, Mazzella M, Arioni C, Campisi C, Taddei G, Tomà P, Boccardo F, Hennekam RC, Serra G. Hennekam syndrome presenting as nonimmune hydrops fetalis, congenital chylothorax, and congenital pulmonary lymphangiectasia. Am J Med Genet $A$. 2003;120A(1):92-6.
15. Bellini C, Boccardo F, Campisi C, Bonioli E. Congenital pulmonary lymphangiectasia. Orphanet J Rare Dis. 2006;1:43.

16. Castagno R, Carreras E, Toran N, Higueras T, Sánchez M, Cabero L. Fetal pleural effusion. DSJUOG. 2007;1:28-39.

17. Rustico MA, Lanna M, Coviello D, Smoleniec J, Nicolini U. Fetal pleural effusion. Prenat Diagn. 2007;27(9):793-9.

18. Yinon Y, Kelly E, Ryan G. Fetal pleural effusions. Best Pract Res Clin Obstet Gynaecol. 2008;22:77-96.

19. Bellini C, Hennekam RC. Clinical disorders of primary malfunctioning of the lymphatic system. Adv Anat Embryol Cell Biol. 2014; 214:187-204.

20. Kim SA, Lee SM, Hong JS, et al. Ultrasonographic severity scoring of non-immune hydrops: A predictor of perinatal mortality. J Perinat Med. 2015;43:53-59.

21. Aubard Y, Derouineau I, Aubard V, Chalifour V, Preux PM. Primary fetal hydrothorax: A literature review and proposed antenatal clinical strategy. Fetal Diagn Ther. 1998;13(6):325-33.

22. Weber AM, Philipson EH. Fetal pleural effusion: a review and meta-analysis for prognostic indicators. Obstet Gynecol. 1992; 79(2):281-6.

23. Klam S, Bigras JL, Hudon L. Predicting outcome in primary fetal hydrothorax. Fetal Diagn Ther. 2005;20(5):366-70.

24. Trocado V, Coutinho-Borges JP, Carlos-Alves M, Santos J, Pinheiro P. Fetal primary hydrothorax with spontaneous resolution. Case Rep Womens Health. 2017;15:6-7.

25. Ylänen K, Poutanen T, Savikurki-Heikkilä P, Uotila J, Korppi M, Eerola A. Bilateral absence of the superior vena cava. Case Rep Cardiol. 2012;2012:461040.

26. del Ojo JL, Delgado Jimenez C, Jimenez Vilches P, Lesmes Serrano A, Lozano Gutierrez F, Beltran Rodriguez J. Absence of the superior vena cava: difficulties for pacemaker implantation. Pacing Clin Electrophysiol. 1999;22(7):1103-5.

27. Quraishi MB, Mufti O, Wase A. Absent left and right superior vena cava and azygos continuation of inferior vena cava: a rare anomaly of systemic venous return. J Invasive Cardiol. 2010;22(8):E159-61.

28. Krasemann T, Kehl G, Vogt J, Asfour B. Unusual systemic venous return with complete absence of the superior caval veins. Pediatr Cardiol. 2003;24(4):397-9.

29. Romer S, Opgen-Rhein B, Chaoui R et al. Congenital absence of superior vena cava associate with high position of aortic arch. Ultrasound in Obstetrics and Gynecology 2006;28(6):842-844.

30. Lee C.Y, Jan S.L, Wang TM et al. Congenital chylothorax associated with isolated congenital hypoplastic superior caval vein: a case report. Acta Paediatrica, International Journal of Paediatrics 2005; 94(12):184-1843.
Conflict of interest: none declared
Financial support: none declared 